\title{
Graphene-based polymer nanocomposites: materials for future revolution
}

\begin{abstract}
The material graphene is always attracting the researchers and scientists' attention by its fascinating properties. Even it has been a decade after its discovery; scientists are still exploring the hidden potentials of this material. Focus on the current research trend in the field of polymer nanocomposites, this review is centered on graphene oxide (GO) and reduced graphene oxide (RGO) based polymer nanocomposites and their potential application opportunities in various emerging areas like carbon dioxide $\left(\mathrm{CO}_{2}\right)$ capture, water purification, photovoltaic cell, sensor, Li-ion batteries, supercapacitors, protective coating, flame retardance composites, electronic devices, automobile body parts, antibacterial coating, biomedical applications (bone and tissue engineering), flexible packaging etc. The aim is to show the full potential of the wonder materials to uplift the standard of living and to judge whether the materials really have the potential to bring future revolution or not.
\end{abstract}

Keywords: graphene oxide (GO), reduced graphene oxide (RGO), nanocomposites, application
Volume I Issue 3 - 2017

\author{
Madhab Bera, Pradip K Maji \\ Department of Polymer and Process Engineering, Indian \\ Institute of Technology Roorkee, India
}

\begin{abstract}
Correspondence: Pradip K Maji, Department of Polymer and Process Engineering, Indian Institute of Technology Roorkee, Saharanpur Campus, Saharanpur-24700I, UP, India,
\end{abstract} Email pradip.fpt@iitr.ac.in

Received: May 02, 2017 | Published: June 13, 2017
Abbreviations: GO, graphene oxide; RGO, reduced graphene oxide; $\mathrm{CO}_{2}$, carbon dioxide; $\mathrm{BHJ}$, bulk-heterojunction; EMI, electromagnetic interference; PVA, polyvinyl alcohol; PEI, polyethylenimine

\section{Introduction}

Materials science is playing an important role in our daily lives, especially in the development of new of materials. Polymer nanocomposite is a result of continuous effort, successfully journey of which was started from 1993 after the pioneer work done by Usuki et al. ${ }^{1}$ Polymer nanocomposite is an engineered material made by dispersing nanofillers (filler having at least one dimension within $1-100 \mathrm{~nm}$ range) within the polymer matrix. It shows astonishing improvement in thermal, mechanical, electrical, barrier, antibacterial etc. properties with the addition of a very little amount of filler, without affecting the density of the material. The discovery of graphene was a major breakthrough in the field of material science, in 2004 by two renowned physicists KS Novoselov et al. ${ }^{2}$ from Manchester University. This discovery started a new era and graphene has becoming the rapidly rising star. The two dimensional (2D) nanomaterial is basically anatomically thick monolayer graphite sheet where every carbon atom is $\mathrm{sp}^{2}$ hybridized and arranged in a hexagonal manner. This type of close pack arrangement makes it extraordinary in strength and the strongest material in the world. It is 100 times stronger than steel with tensile strength of $130 \mathrm{GPa}$ and tensile modulus of $1 \mathrm{TPa}$. Graphene has also an extremely high specific surface area (theoretical value $\left.2630 \mathrm{~m}^{2} / \mathrm{g}\right)$, excellent thermal conductivity $\left(5000 \mathrm{Wm}^{-1} \mathrm{~K}^{-1}\right)$ and electrical conductivity $\left(200,000 \mathrm{~cm}^{2} \mathrm{~V}^{-1} \mathrm{~S}^{-1}\right) .{ }^{3}$ However, due to its very hydrophobic nature and high surface energy, the raw graphene is seldom compatible with almost all available organic polymers and can't be dispersed uniformly. So, uniform dispersion of graphene sheets within the polymer matrix is a big challenge to the researchers. Chemical modification or functionalization is the only way to make its stable suspension and uniform dispersion. In this context, the use of graphene based materials like graphene oxide (GO) and reduced graphene oxide
(RGO) are smart alternative choices. GO is synthesized from low cost and available graphite. It contains large number of oxygen containing functional groups like hydroxyl, carbonyl, carboxyl and epoxy which make it compatible with most of the organic polymers. RGO is obtained by simple reduction of GO, in a variety of methods (thermal, chemical, hydrothermal etc.). It contains very less number of oxygen containing functional groups which make it compatible with very less polar or non polar organic polymers like polyethylene, polystyrene etc. The first report on graphene based polymer nanocomposite was published in 2006 by utilizing polystyrene and graphene. After that a wide range of polymers have been used for synthesizing graphenepolymer nanocomposites according to the diversified demand of modern technologies. This review is inclined more towards GO and RGO based polymer nanocomposites and their applications in diversified areas which can bring future revolution.

\section{Discussion}

Two kinds of interactions exists between graphene based materials and polymer matrix, covalent and non covalent. In case of covalent bonding interaction, a permanent covalent bond is formed between polymer and graphene based materials. Whereas, for non-covalent bonding interaction, $\pi-\pi$ and electrostatic interaction exists between polymer and filler (Figure 1A). Based on these interactions, graphene based polymer nanocomposites have three different structures: phase separated, intercalated and exfoliated (Figure 1B). Excellent properties of graphene based polymer nanocomposites originating from these different kinds of interactions, endows its applications in photovoltaic cells,${ }^{4,5}$ supercapacitors ${ }^{6}{ }^{6} \mathrm{Li}$ ion batteries, ${ }^{7}$ electronic devices, protective coating, antibacterial coating and composites, ${ }^{8}$ fire retardant composites, ${ }^{9,10}$ biomedical applications, sensors, water purification, carbon dioxide capture, ${ }^{11}$ etc.

\section{Photovoltaic cells}

It is estimated that global energy demand will be almost doubled within next 50 years. Fossil fuel stock is running out. Further, the 
energy generated by utilizing this source creates a lot of environmental pollution. Hence, development of an environmentally friendly, clean and renewable energy technology has been raised to a major concern in the $21^{\text {st }}$ century. Almost $90 \%$ of the photovoltaic cells available in the market are based on a semiconductor material, silicon $(\mathrm{Si})$, which have higher power conversion efficiency (18-24\%) but are very costly and have limited availability. Use of conjugated organic polymers like polyaniline can solve these issues because they are low cost, easily available, light weight and flexible. Because of high electrical conductivity (max. value $200,000 \mathrm{~cm}^{2} \mathrm{~V}^{-1} \mathrm{~S}^{-1}$ ), high carrier mobility and moderately high optical transparency $(\sim 97 \%)$ graphene based polymer nanocomposite materials can be utilized in dye-sensitized solar cells and bulk-heterojunction (BHJ) which can increase the power conversion efficiency up to $10-15 \%$ at lower cost. ${ }^{4,5}$
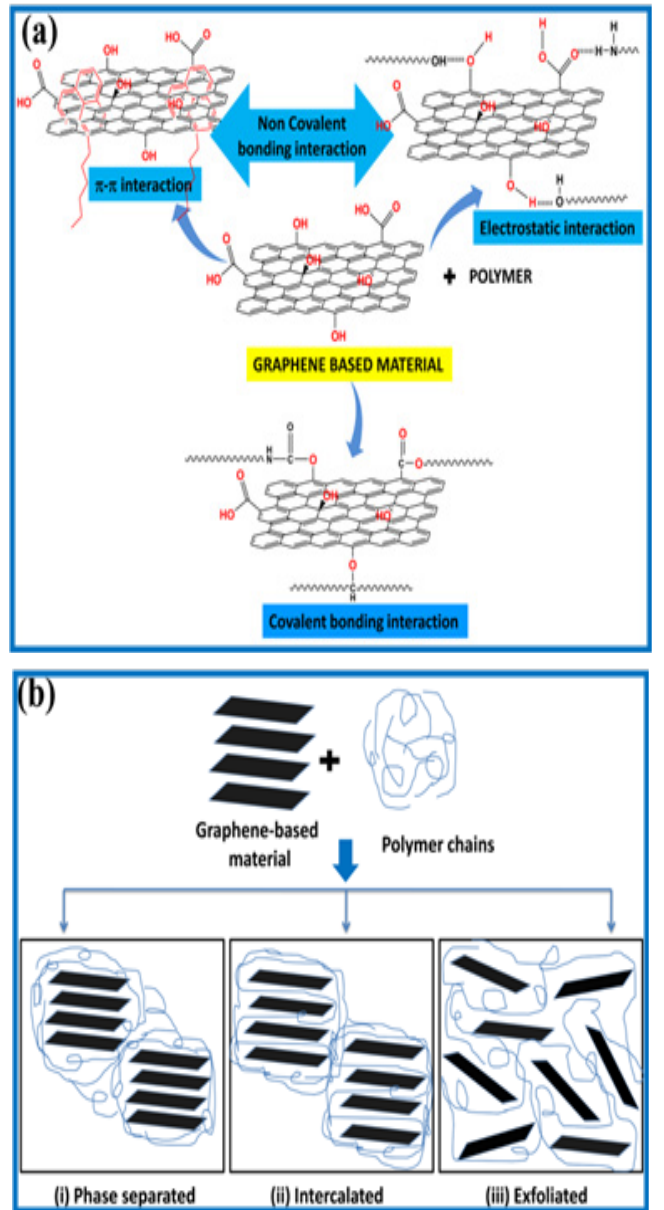

Figure I Schematic representation of

(A) Different kind of interactions (covalent and non-covalent) between graphene based materials and polymer matrix.

(B) Different type of structures of graphene-based polymer nanocomposite: (i) phase separated (ii) intercalated and (iii) exfoliated.

\section{Battery and supercapacitors}

Battery and supercapacitors are two other energy related application areas where graphene based polymer nanocomposite can be utilized. Graphene based polymer nanocomposite brings the revolution of light weight and high capacity battery and supercapacitors. Li et al. ${ }^{6}$ reported the fabrication of flexible solid-state supercapacitor base on
GO and polyaniline with specific capacitance of $1095 \mathrm{Fg}^{-1}$ at $1 \mathrm{Ag}^{-1}$. Graphene based polymer nanocomposites become a suitable option as an electrode material for Li-ion batteries also. ${ }^{7}$

\section{Electromagnetic interference (EMI)}

The intrinsic high thermal and electrical conductivities of graphene based polymer nanocomposites endows its application in Electromagnetic interference (EMI) shielding application. EMI is an important and familiar terminology for electronics, telecommunication and instrumentation sector. It refers to the blocking of electromagnetic radiation arising from other nearby appliances. This property is very much essential in designing the instrument because the performance of the instrument depends on the EMI shielding efficiency of individual component inside the complex instrument. Graphene/ polymer nanocomposites can also be utilized to improve the EMI shielding efficiency of the electronic components and is an emerging area of research.

\section{Shape memory application}

Shape memory property of polymer nanocomposite is very crucial for their application in variable atmospheres, like change in temperature, light intensity, stress, magnetic and electric field or solutions. Graphene based polymer nanocomposites can be used for this purpose which finds its application in robotics, orthopedic surgery, self-repairing structural components like automobile fenders in which dents are repaired by application of temperature, biomedical applications etc. A range of polymers can be used together with different types of graphene based materials for different shape memory applications. For example graphene/vitrimer nanocomposites are used for thermally induced shape memory application, thermoplastic polyurethane/ isocyanate modified reduced graphene oxide (iTRG) is used for electroactive shape memory application, water induced shape memory effect was observed with polyvinyl alcohol (PVA) reinforced by graphene oxide. UV light induced shape memory effect of anthracene is also a major area of research.

\section{Antibacterial composites}

Most of the research activities on improving the antibacterial property of materials are mainly directed towards the utilization of some metal nanoparticles like $\mathrm{Ag}, \mathrm{Au}$ and metal oxides like $\mathrm{ZnO}$ and $\mathrm{TiO}_{2}$. It is Liu et al. ${ }^{8}$ who reported the antibacterial activity of GO and RGO towards Escherichia coli bacterial. Superior antibacterial activity of GO was observed. Also, the antibacterial activity of these materials increases with increase in concentration of aqueous solution and incubation time. Again, antibacterial capacity is dependent on particle size also; the smaller the size the greater is the antibacterial property. Till now antibacterial activity of different combination of polymer/graphene based materials has been investigated. For example, poly-N-vinyl carbazole (PVK)/GO antimicrobial film, PLA/ PU/GO ternary composites, silver nanoparticle decorated GO (GO$\mathrm{Ag}$ )/PVDF nanocomposite etc. But, very little work has been done on polyurethane/graphene combination. So, it's a great area of research. These types of nanocomposites find promising application in the tissue engineering sector and different types of coating and packaging.

\section{Flexible packaging}

Flexible packaging is also an important area which can be explored. Incorporation of graphene to the polymer matrix improves the thermal, mechanical and barrier properties to a great extent. 
Further, the antibacterial or antimicrobial activity of graphene based materials can also be exploited for this purpose. Polylactic acid (PLLA)/graphene nanocomposite is one such example which can be used in bio based food packaging application. Many other examples are also available like LLDPE/EVA/graphene, GO nanosheets/poly (vinyl alcohol) nanocomposite. This is also a growing field where many other combinations of polymer and graphene based materials can be tried.

\section{Protective coating}

Protective coating is another growing area where graphene based polymer nanocomposites can be utilized, because graphene has excellent chemical resistance, gas impermeability, antibacterial properties, mechanical strength, lubricity and thermal stability. Hence, it can be modified and mixed with various types of polymers for synthesizing self cleaning and corrosion barrier coatings.

\section{Flame retardance applications}

From the last few decades the use of organic polymers and their composites in our daily life increases tremendously because of their various advantages. Now-a-days their use is ubiquitous. However, one of the major drawbacks of organic polymers is that they are intrinsically flammable. So, reduction of flammability is a major concern of the researchers and scientist, especially for electrical application where decreased flammability is demanded .Various works have been done to increase flame retardance properties of polymer by incorporating montmorillonite clay. ${ }^{9}$ Recently, G Huang et al. ${ }^{10}$ shown from cone calorimeter experiment that PVA/graphene nanocomposite shows better flame retardance property than MWNT or MMT filled nanocomposites. According to them flame retardance property of PVA/graphene nanocomposite increases with increase in graphene content. So, graphene based polymer nanocomposites can find a commanding position in different applications like protective coating, flexible packaging, automobile industries etc.

\section{Biomedical applications}

Polymer nanocomposites containing graphene based materials and biodegradable polymers like poly (lactic acid), poly (caprolactone), poly ( $\mathrm{N}$-vinylcarbazole) (PVK) etc. have proven to be a potential candidates for biomedical applications because of their high surface area, excellent toxicity towards bacteria and low cytotoxicity to human cells. One of the most important and growing area is tissue engineering, where composite scaffold is being used to cure the damaged tissues. Ever increasing number of publications in the $\mathrm{SCI} / \mathrm{Scopus}$ database has proven that the field is growing. Different types of scaffolds are used for different applications. For example, electrospinning thermoplastic polyurethane/graphene oxide scaffolds for small diameter vascular graft tissue engineering application, biodegradable porous polyurethane scaffolds for tissue repair and regeneration, GO/PVA nanocomposite scaffolds for bone tissue engineering. Other potential applications are in drug delivery, gene therapy and cancer therapy.

Application of graphene based materials in sensors is an emerging area of biomedical research. The basic principle of a sensor is the change of conductance as a function of the extent of surface adsorption. Large specific area and low Johnson noise, graphene has been proved to be a promising candidate to detect a variety of molecules such as gases to bio-molecules. Furthermore, its applications can be extended to $\mathrm{pH}$, pressure and temperature sensors.

\section{Water purification}

Water and fresh air become an indispensable part of our life. With the development of civilization and industrialization the availability of pure drinking water becoming very less. Various dyes, unwanted toxic chemicals and minerals extracted from the industries are snatching the pure water day by day. So, it's an alarming concern for the scientists to serve pure and healthy water to everyone. Various research initiatives have been taken to sought out this issue. Research in this line is growing rapidly. GO/polyethylenimine (PEI) hydrogels can be efficiently utilized as an adsorbent of organic dye for waste water. Further, graphene polymer nanocomposites can be used for the removal of lead (II), chromium (VI) etc toxic metal ions from waste water. Graphene based polymeric nanocomposite membrane is another important topic of intense research align to this area. Recently, polysulfone/graphene and cellulose/graphene composite membrane are used in water purification process.

\section{Carbon dioxide capture}

The ever increasing percentage of carbon dioxide $\left(\mathrm{CO}_{2}\right)$ in the atmosphere becomes a serious global issue because it is a major contributor to global warming. Carbon dioxide management in the earth atmosphere is a major field of research. Scientists and researcher all over the world are working on it to find out an economical way to capture the excess $\mathrm{CO}_{2}$ and make a balance of its concentration in the earth atmosphere. Various porous materials are being used to capture $\mathrm{CO}_{2}$, one important addition in this is graphene and graphene based materials. Porous composite aerogel made with biodegradable polymer like chitosan and GO can be used for this purpose. ${ }^{11}$

\section{Conclusion}

Graphene-based polymer nanocomposites are one of the most auspicious developments in the field of material science. In this review, potential applications of graphene-base polymer nanocomposites materials, based on GO and RGO has been highlighted. The three major challenges of the upcoming future are fresh air, pure water and green energy. The use of graphene based polymer nanocomposite materials can solve all these three problems. Hence, we can say undoubtedly that these are the materials for future revolution.

\section{Acknowledgements}

None.

\section{Conflict of interest}

The author declares no conflict of interest.

\section{References}

1. Usuki A, Kojima Y, Kawasumi M, et al. Synthesis of nylon 6-clay hybrid. J Mater Res. 1993;8(5):1179-1184.

2. Novoselov KS, Geim AK, Morozov SV, et al. Electric field effect in atomically thin carbon films. Science. 2004;306(5696):666-669.

3. Zhu Y, Murali S, Cai W, et al. Graphene and graphene oxide: synthesis, properties, and applications. Adv Mater. 2010;22(35):3906-3924.

4. Singh E, Nalwa HS. Stability of graphene-based heterojunction solar cells. RSC Adv. 2015;5(90):73575-73600.

5. Hsu YC, Tseng LC, Lee RH. Graphene oxide sheet-polyaniline nanohybrids for enhanced photovoltaic performance of dye-sensitized solar cells. J Polym Sci Part B Polym Phys. 2014;52(4):321-332. 
6. Li H, Song J, Wang L, et al. Flexible all-solid-state supercapacitors based on polyaniline orderly nanotubes array. Nanoscale. 2017;9(1):193-200.

7. Song Z, Xu T, Gordin ML, et al. Polymer-graphene nanocomposites as ultrafast-charge and-discharge cathodes for rechargeable lithium batteries. Nano Lett. 2012;12(5):2205-2211.

8. Liu S, Zeng TH, Hofmann M, et al. Antibacterial activity of graphite, graphite oxide, graphene oxide, and reduced graphene oxide: membrane and oxidative stress. ACS nano. 2011;5(9):6971-6980.
9. Kashiwagi T, Harris RH, Zhang X, et al. Flame retardant mechanism of polyamide 6-clay nanocomposites. Polymer. 2004;45(3):881-891.

10. Huang G, Gao J, Wang X, et al. How can graphene reduce the flammability of polymer nanocomposites. Mater Lett. 2012;66(1):187-189.

11. Alhwaige AA, Agag T, Ishida $\mathrm{H}$, et al. Biobased chitosan hybrid aerogels with superior adsorption: role of graphene oxide in $\mathrm{CO}_{2}$ capture. $R S C$ Adv. 2013;3(36):16011-16020. 